pecial Reviews

Juntendo Medical Journal

2017. 63 (1), 8-16

\title{
Pathophysiology of Patients with Schizophrenia: Genetic and Environmental Factors
}

\author{
TOHRU OHNUMA*, HEII ARAI* \\ *Juntendo University Schizophrenia Projects (JUSP), Department of Psychiatry, \\ Juntendo University Faculty of Medicine, Tokyo, Japan
}

\begin{abstract}
Schizophrenia is a debilitating disorder with a prevalence of approximately $0.5 \%-1 \%$ within any given population. The pathophysiology of schizophrenia involves complex genetic, environmental, and psychological etiologies. Here we summarize 26 years of research completed by the Juntendo University Schizophrenia Projects study group on the "biopsychosocial model" of schizophrenia. Clinical brain morphological abnormalities in schizophrenia were detected with magnetic resonance imaging, and these findings led to gene expression analyses of neurotransmitters. The familial aggregation pattern in schizophrenia led to the completion of genetic studies, including linkage and genome-wide analyses, and studies on environmental factors, such as nutrition, aging, stress, and inflammation. Furthermore, we developed a collaborative multicenter study that consisted of a large number of samples. This study enabled us to clearly identify the relevant pathophysiology of schizophrenia, including genetics, altered neurotransmission, brain morphology, and clinical features.
\end{abstract}

Key words: schizophrenia, genetics, environment, brain, neurotransmitter

\section{Introduction}

While completing bedside learning during my fifth year of medical school, I met a 27-year-old female patient. She was suffering from slandering auditory hallucinations that were resistant to antipsychotic medications prescribed since the onset of her condition at 17 years of age. Unfortunately, approximately $20 \%-30 \%$ of patients with schizophrenia have a treatment-resistant form of the disorder ${ }^{1)}$. After I became aware of the existence of debilitating symptoms, such as auditory hallucinations, and the $1 \%$ prevalence rate for schizophrenia in the general population, I chose to become a psychiatrist.

\section{Brain morphology}

Schizophrenia is a debilitating disorder with a prevalence of approximately $0.5 \%-1 \%$ within any given population. The pathophysiology of schizophrenia involves complex genetic, environmental, and psychological etiologies. Clinically, atypical brain morphological abnormalities are often observed in patients with schizophrenia using computed tomography (CT) and magnetic resonance imaging (MRI). Previous studies showed that patients with brain morphological abnormalities typically have a severe form of the disorder that is relatively resistant to treatment ${ }^{2)}$. However, it was unclear if these atypical brain morphological abnormalities existed prior to symptom onset or appeared as the disorder progressed. To answer this question, I conducted an MRI study on patients

Corresponding author: Tohru Ohnuma

Juntendo University Schizophrenia Projects (JUSP), Department of Psychiatry, Juntendo University Faculty of Medicine

2-1-1 Hongo, Bunkyo-ku, Tokyo 113-8421 Japan

TEL: +81-35802-1071 (ext. 3319) E-mail: otoru@juntendo.ac.jp

339th Triannual Meeting of the Juntendo Medical Society: Medical Research Update〔Held on May 21, 2016〕

[Received July 15, 2016]

Copyright (C) 2017 The Juntendo Medical Society. This is an open access article distributed under the terms of Creative Commons Attribution License (CC BY), which permits unrestricted use, distribution, and reproduction in any medium, provided the original source is properly credited. doi: $10.14789 /$ jmj. 63.8 


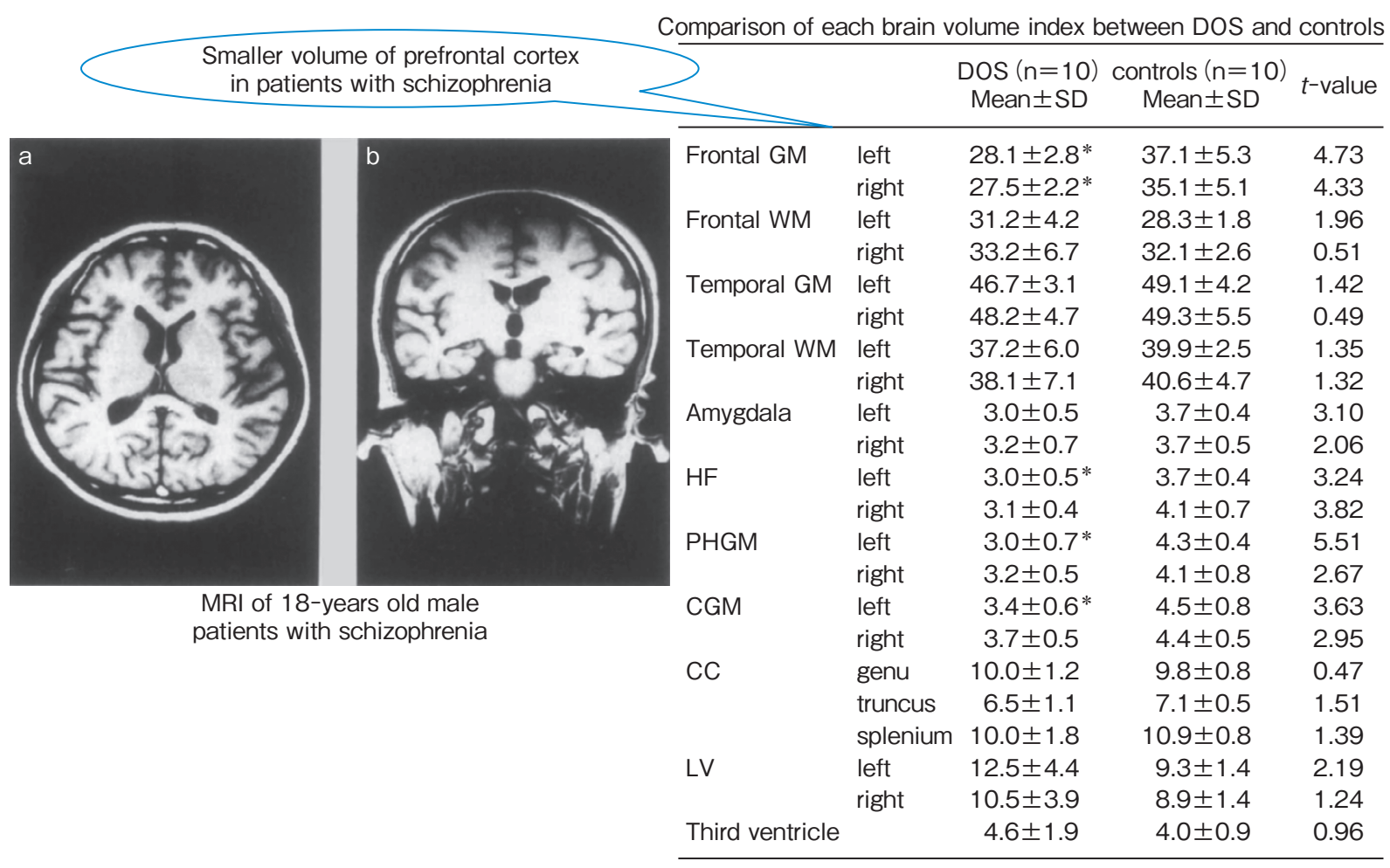

*p<0.002 ; GM, gray matter ; WM, white matter ; HE, hippocampal formation ; PHGM, parahippocampal gyrus gray matter ; CGM, cingulate gyrus gray matter; CC, corpus callosum.

Figure-1 A quantitative magnetic resonance imaging study revealed that patients with schizophrenia had a small prefrontal cortex (Ohnuma T, et al: Psychiatry Clin Neurosci, 1997; 51, partial modification ${ }^{3)}$ )

with schizophrenia during their first episodes, prior to neuroleptic treatment. These patients were found to have small prefrontal and temporal cortices as well as a reduced hippocampus volume at symptom onset, which supports a neurodevelopmental hypothesis (Figure-1) ${ }^{3)}$. Thus, we could not refer to these findings as "atrophy" because the detailed neuropathological examination of the brains from individuals with schizophrenia failed to reveal any prominent neuronal loss or typical neurodegenerative changes (e.g., gliosis) ${ }^{4)}$ ).

\section{Post-mortem brain study}

To further investigate the genesis of morphological changes of the brain, including gene expression levels, I worked with the Babraham Institute (Medical Research Council, Laboratory of Molecular Neuroscience Group, Department of Neurobiology) at Cambridge University from 1996 to 1997 as a visiting scholar (Figure-2). I learned several molecular biology techniques, including Northern and Southern blots, in-situ hybridization, and gene

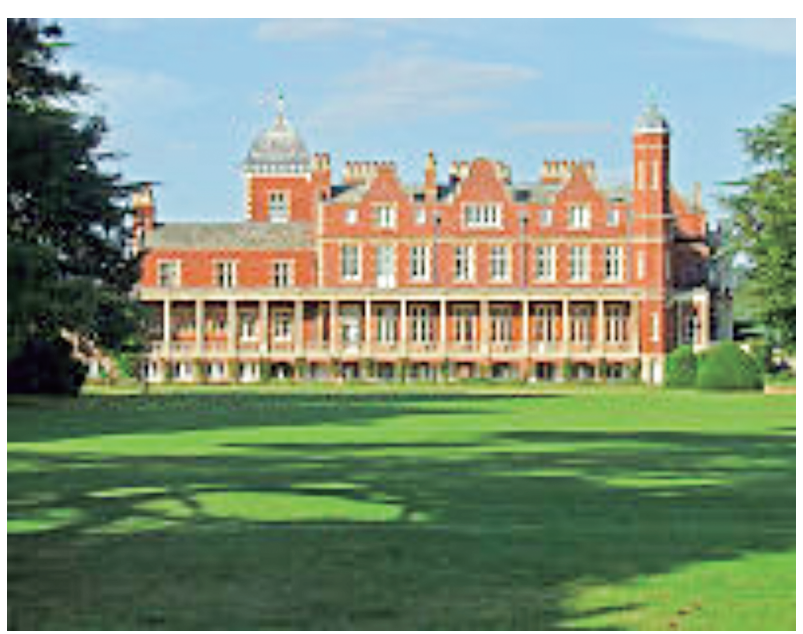

Figure-2 The Babraham Institute in Cambridge, United Kingdom

targeting in mice. I used these methods to determine the concentration and gene expression of several neurotransmitters in the prefrontal and temporal cortices of fresh-frozen post-mortem brains of patients with schizophrenia from Cambridge University Hospital. First, I investigated glutamate levels as well as the mRNA expression of 


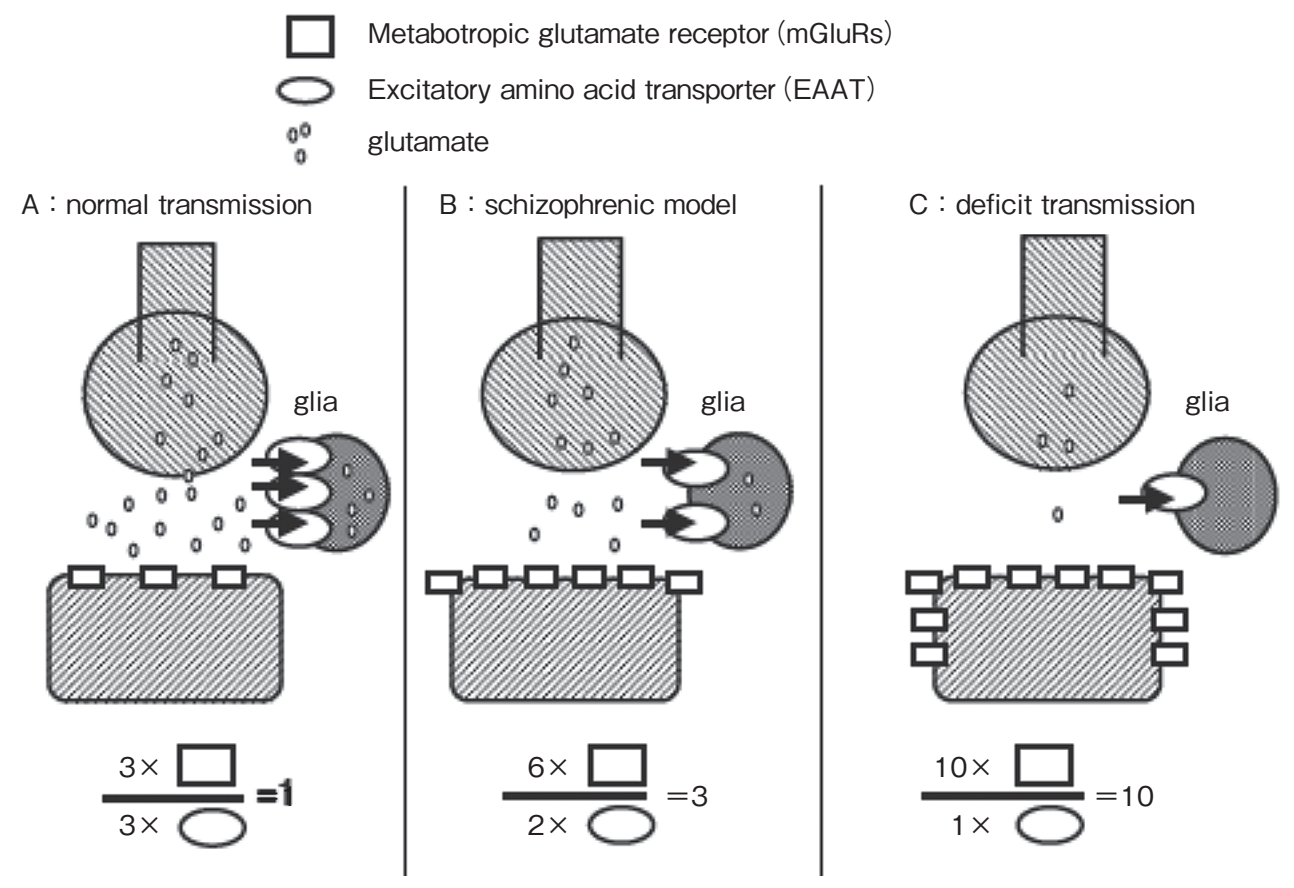

Figure-3 A model of the synaptic cleft in patients with schizophrenia (Ohnuma, et al: Prog Neuropsychopharmacol Biol Psychiatry, 2005; $29^{8)}$ )

glutamate receptors and transporters in the brains of patients with schizophrenia and healthy controls to detect any changes in glutamatergic neurotransmission at the synaptic cleft. There was a tendency toward increased metabotropic glutamate receptors (mGluRs) and decreased excitatory amino acid transporter 2 (EAAT2) mRNA in all of the prefrontal areas examined; however, no significant differences were observed between patients with schizophrenia and controls. To better assess minimal changes in glutamatergic neurotransmission at the synaptic cleft, the "receptor/transporter ratio" (i. e., mGluRs/EAAT2 ratio) was calculated. The mGluRs/EAAT2 ratio was significantly higher in patients with schizophrenia than in controls. While measured glutamate levels using HPLC showed a non-significant decrease in postmortem schizophrenic brain samples ${ }^{6)}$. Similar results were found for $\mathrm{GABA}^{7)}$. Overall, these data suggest that the increase in mGluRs and decrease in EAAT2 mRNA could be a compensatory mechanism for the decrease in glutamate transmission at the synaptic cleft in schizophrenic brains. However, these changes were small and not statistically significant. The "receptor/transporter ratio" combined these individual changes to produce statistically significant results $(\text { Figure }-3)^{8}$. Thus, we hypothesized that minimal pathological changes in the prefrontal cortex, such as decreased glutamatergic and GABAergic neurotransmission in patients with schizophrenia.

\section{Juntendo University Schizophrenia Projects}

In 2003, the Juntendo University Schizophrenia Projects (JUSP) study group was established in our department. The aim of JUSP is to resolve the complicated and multifactorial pathophysiology of schizophrenia based on a biopsychosocial model (Figure-4).

\section{Genetic studies}

The JUSP study group completed several genetic case-controlled studies to identify genes involved in the pathophysiology of schizophrenia ${ }^{9)-18)}$. Positive findings from JUSP studies were reassessed in a large-scale genetic replication study (e.g., approximately 2,500 patients with schizophrenia and 2,500 controls) by collaborative centers in Japan ${ }^{19)-22)}$. Despite their strong statistical power, these replication analyses failed to show any significant genetic associations with schizophrenia. However, these results suggested that clinically-observed familial aggregation in patients with schizophrenia cannot 


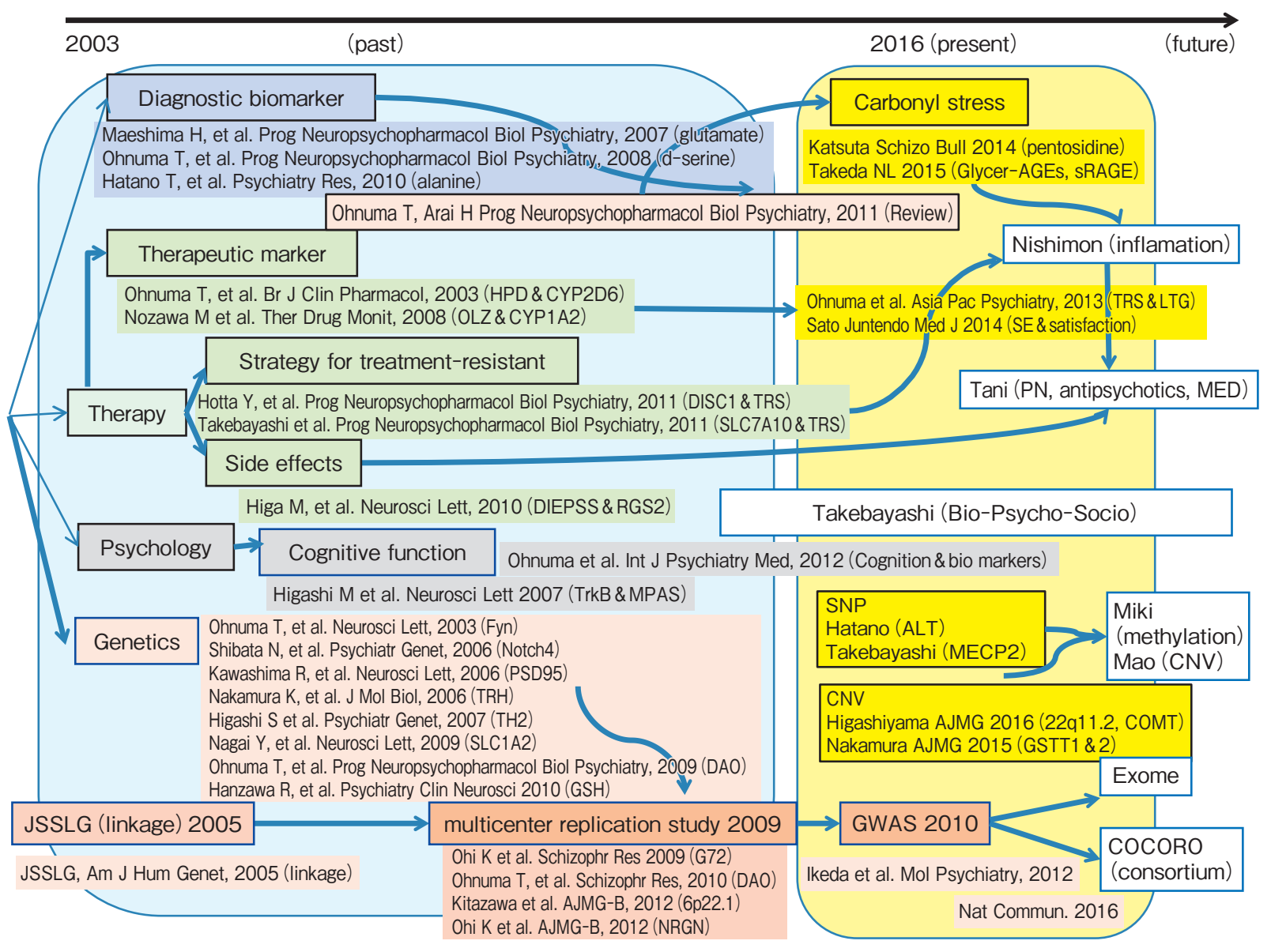

Figure-4

The relationships among the studies completed under the Juntendo University Schizophrenia Projects (JUSP) study group since 2003.

be explained by the common disease-common variant hypothesis. Strong familial aggregation, which was observed in some families, is not always caused by genetic factors. Indeed, a large linkage analysis of 236 Japanese schizophrenia familial aggregation, including JUSP samples from the Japanese Schizophrenia Sib-Pair Linkage Group, supports the existence of schizophrenia susceptibility loci on chromosomes $1 \mathrm{p}, 14 \mathrm{q}$, and $20 \mathrm{p}^{23)}$. However, these results were not reproduced in replication studies with other ethnic populations. Thus, some environmental factors (e.g., food and stress) are likely to play a role in schizophrenia. Strong psychological sympathy can result in transmitted psychosis termed "folie à deux," and the development of schizophrenia-like symptoms and high familial aggregation ${ }^{24)}$. These results led our research group to complete a genome-wide association study (GWAS) while also investigating biological environmental factors.

\section{Peripheral biomarker studies}

We reported that hypoglutamatergic neurotransmission occurs in the brains of patients with schizophrenia. To look for biological markers of schizophrenia, we studied several excitatory amino acids involved in glutamate neurotransmission via NMDA receptors, such as endogenous glutamate, glycine, serine, and alanine. We reviewed several studies of excitatory amino acid levels in peripheral blood and our previous JUSP biomarker studies, which thoroughly investigated plasma glutamatergic amino acid levels ${ }^{25)-28}$, to determine if any of these amino acids could be used as diagnostic, therapeutic, or symptomatic biological markers ${ }^{29)}$. We concluded that peripheral blood levels of endogenous glycine and alanine may be symptomatic markers for schizophrenia, whereas levels of exogenous glycine and alanine may be therapeutic markers. Notably, peripheral blood levels of endogenous D-serine may correlate with its brain concentration, which suggests that this amino acid may be 
A

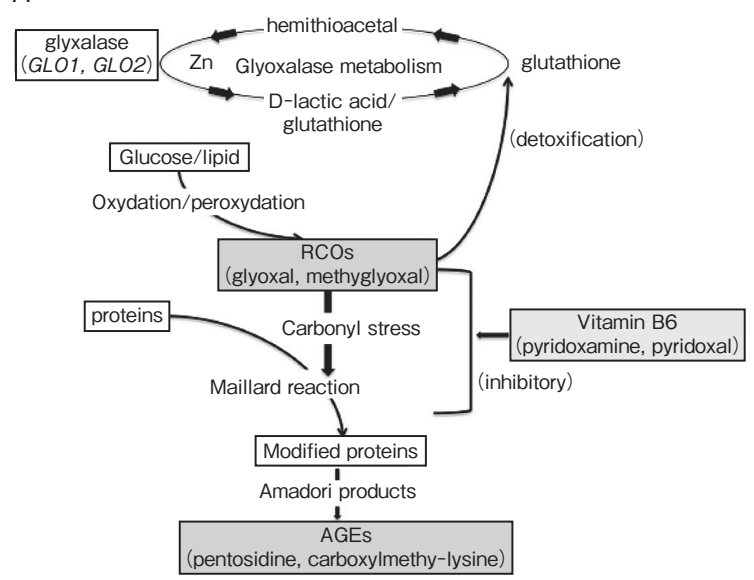

B1

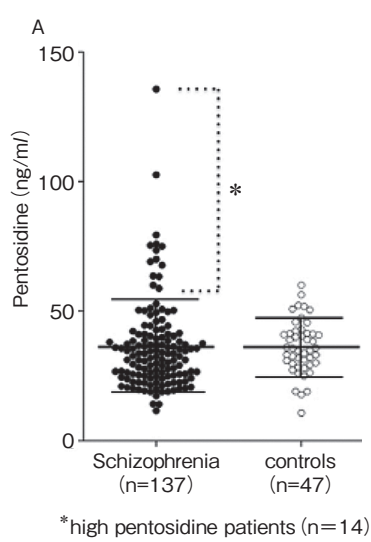

B2

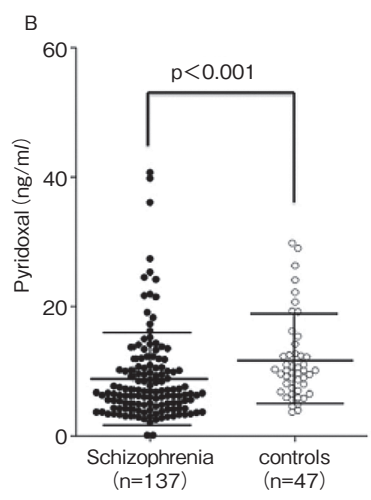

Figure-5

A. The mechanism of carbonyl stress and its detoxification with vitamin B6 and glyoxalase Reactive carbonyl compounds (RCOs) cause carbonyl stress; however, RCOs can be detoxified by degradation into lactic acid and glutathione by glyoxalase enzymes. Glyoxalase 1 and 2 (GLO1 and GLO2) are the rate-limiting enzymes in this metabolic pathway. Inhibition of RCO generation and the Maillard reaction by vitamin B6 results in the suppression of AGE accumulation.

B. Serum levels of carbonyl stress markers in normal controls and patients with schizophrenia at hospital admission

B1. Pentosidine. B2. Pyridoxal. Fourteen patients with high pentosidine levels $(>2 \mathrm{SD}$ above the mean of controls, i.e., $>57.6 \mathrm{ng} / \mathrm{m} l)$ are indicated with an asterisk. Values were compared using a two-tailed Mann-Whitney $U$ test. Data are shown as the mean \pm SD.

a useful diagnostic and therapeutic marker for schizophrenia ${ }^{29)}$.

In 2010, a cross-sectional study of chronic schizophrenia reported altered peripheral carbonyl stress markers, including high levels of serum pentosidine that accumulates following carbonyl stress and low levels of pyridoxal (vitamin B6) that detoxifies reactive carbonyl compounds ${ }^{30)}$. In crosssectional and longitudinal studies, we investigated if the serum levels of these markers reflected the clinical course of the disorder ${ }^{31}$. One hundred and thirty-seven acute-stage Japanese patients were enrolled. Among these, 53 patients were followed from the acute stage to remission. A portion of patients in the acute stage (14 cases, 10.2\%) showed extremely high pentosidine levels (Figure-5). These levels were not associated with the severity of symptoms, but were associated with antipsychotic dose amounts. Pyridoxal levels were lower in schizophrenia and increased according to the clinical course of the illness (Figure-5). Furthermore, 18 patients with decreased pyridoxal levels according to the clinical course showed that the greater the decrease in pyridoxal levels, the less improvement in symptoms. Thus, extremely high pentosidine levels in a portion of patients may be caused by higher daily antipsychotic doses, whereas pyridoxal levels were lower in schizo- phrenia and increased according to the clinical course. Patients with decreasing pyridoxal levels during the clinical course showed less improvement in symptoms. Carbonyl stress markers may also be therapeutic biological markers in some patients with schizophrenia ${ }^{31)}$. We measured glyceraldehyde-derived AGEs (Glycer-AGEs), which are highly neurotoxic, and soluble receptors for AGEs (sRAGE), which may ameliorate the effects of AGEs, to determine their potential as diagnostic, therapeutic, or clinical biological markers in patients with schizophrenia ${ }^{32}$. Glycer-AGEs were significantly higher and sRAGE levels were significantly lower in patients with acute schizophrenia compared with those in healthy controls (Figure-6). Furthermore, discriminant analyses confirmed that Glycer-AGE levels and Glycer-AGEs/sRAGE ratios can be used as diagnostic markers for schizophrenia and distinguished between patients and healthy controls in $70.0 \%$ of cases. These data highlight the potential of Glycer-AGEs, sRAGE, and their relative ratios as diagnostic markers for patients with schizophrenia ${ }^{32)}$. Based on the speculation that aging stress advances inflammation, we are further investigating micro-inflammation biomarkers in patients with schizophrenia. 
A

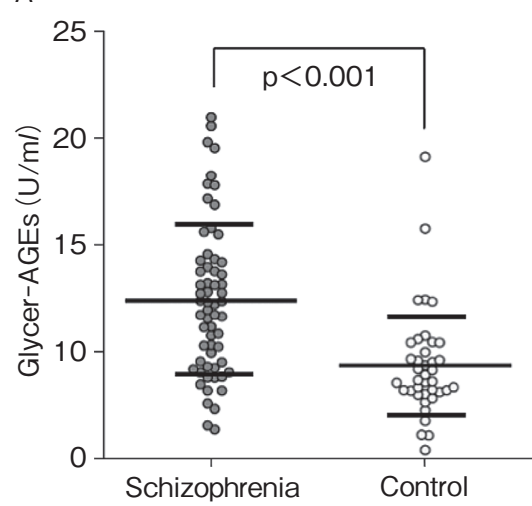

B

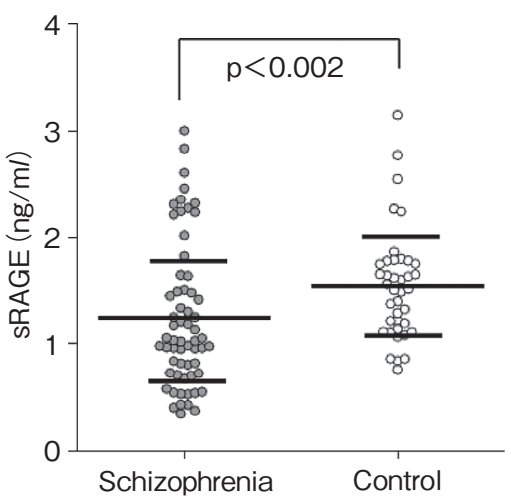

C

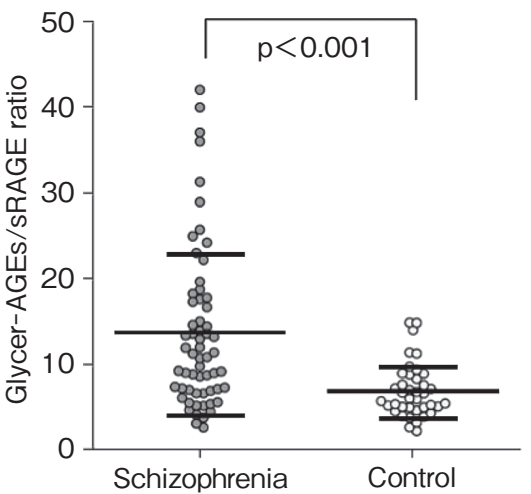

Figure-6 Serum levels of carbonyl stress markers at hospital admission

Glycer-AGEs levels (A), sRAGE level (B), and Glycer-AGEs/sRAGE ratio (C) were compared between patients with schizophrenia and healthy control subjects using a two-tailed Mann-Whitney $U$ test. Data are presented as the mean \pm SD.

\section{Collaborative, large-scale multicenter study}

\section{Genome wide association study}

To test the common disease-common variant hypothesis of schizophrenia, a GWAS was completed as part of our collaborative multicenter study ${ }^{33}$. Significant genome-wide differences were detected in the meta-analysis of the combined data sets $(6,668$ patients and 12,791 controls) from the Japanese population, including JUSP samples $(\mathrm{p}=$ $\left.3.4 \times 10^{-8}, \mathrm{OR}=0.87,95 \% \mathrm{CI}=0.83-0.92\right)$. Assuming a prevalence of $1 \%$, these results suggest that in a population of 700 without risk $\mathrm{T}$ allele of the rs2071287 SNP in NOTCH4, there should be seven patients with schizophrenia. In a population of 700 with at least one risk $\mathrm{C}$ risk allele, there should be eight patients with schizophrenia with an OR of 1.15. Thus, genetics can weakly influence the pathophysiology of schizophrenia via common SNPs.

\section{Copy number variations}

Based on the etiological limitations of the common disease-common variant hypothesis for schizophrenia, genetic studies of copy number variations $(\mathrm{CNV}) \mathrm{s}$, mainly rare CNVs, were developed ${ }^{34)}{ }^{35}$. Indeed, subjects with rare large deletions $(>3 \mathrm{Mb})$ of the ch22q11.2 region (22q11.2 deletion syndrome) often show schizophrenia-like symptoms ${ }^{36}$. Relatively large CNVs $(>1 \mathrm{Mb})$ that account for $50 \%$ of all CNVs in humans are considered to be de novo CNVs. In other words, these CNVs are not inherited and the effects of these mutations would not be reflected in the aforementioned linkage and GWAS studies. Common CNVs (frequencies> $0.05)$, especially those in the ch22q11.2 region should be investigated in patients with schizophrenia. We previously completed a CNV case-control study for $C O M T^{37)}$ and the glutathione (GSH)related genes GSTT1 and GSTT2 ${ }^{38)}$. Our second multicenter replication study included a large number of subjects (1,854 patients vs. 2,137 controls) revealed significant association between CNVs at the promoter 1 and exon 6 regions of COMT and the presence of schizophrenia ${ }^{37}$. These results highlight the importance for further $\mathrm{CNV}$ analyses that address specific $\mathrm{CNV}$ characteristics, such as de novo/inherited or large/small CNVs.

\section{Is schizophrenia a syndrome? Biopsychosocial modeling in a subpopulation of patients with schizophrenia}

A recent collaborative multicenter study reported interesting results ${ }^{39)}$ (Figure-7). This study had three major findings: 1) the risk allele of a brain-enriched sorting nexin, ARHGAP33 (a highaffinity receptor for brain-derived neurotrophic factor), was associated with patients with schizophrenia; 2) ARHGAP33 knockout mice exhibited reduced synaptic $T r k B$ expression, impaired spine development, and neuropsychiatric disorderrelated behavioral abnormalities; and 3 ) the risk allele of ARHGAP33 is associated with smaller prefrontal and temporal cortical volumes based on MRI measurements from patients with schizophrenia ${ }^{3)}$. Thus, this study shows the sequential effects 
A

ARHGAP33

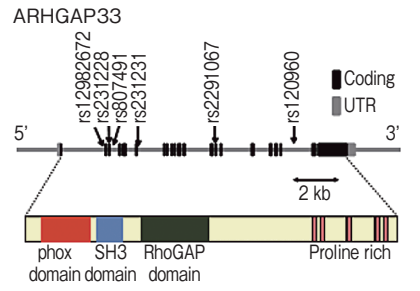

C $\square$ C carrier $\square$ Risk-T/T carrier

Right medial frontal gyrus Right inferior temporal gyrus Left middle temporal gyrus

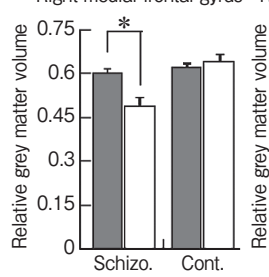

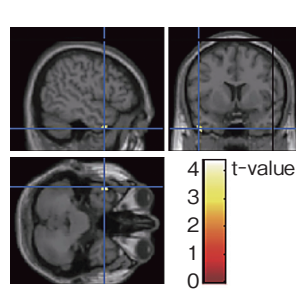

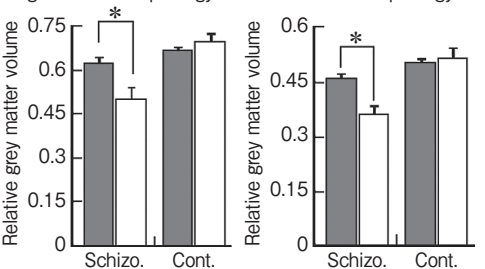

D

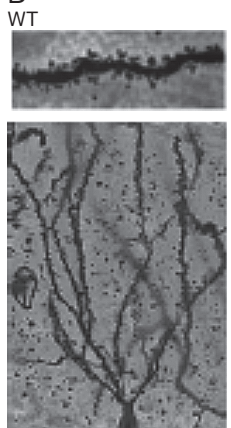

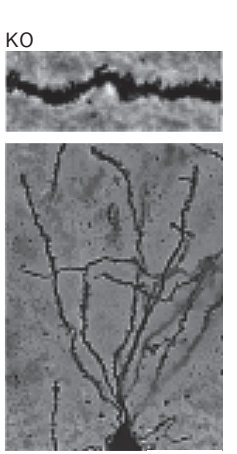

E

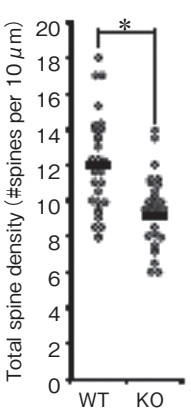

$\mathrm{F}$

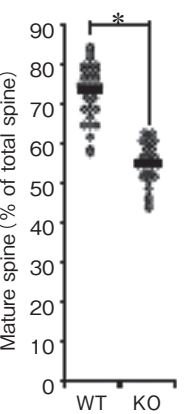

Figure-7 ARHGAP33 is associated with schizophrenia, and ARHGAP33 KO mice show impaired spine development A. The locations of the SNPs analyzed in this study. B. and C. The impact of the risk T allele on grey matter volume of the left middle temporal gyrus in patients with schizophrenia. A significant cluster of the genotype effect was observed in the left middle temporal gyrus of patients with schizophrenia. The regions are identified by cross-hairs. The relative grey matter volumes were extracted from the left middle temporal gyrus, right medial frontal gyrus and right inferior temporal gyrus. ${ }^{*} \mathrm{p}<0.05$. Data are expressed as the mean \pm SEM. D. ARHGAP33 KO mice showed a decrease in total and mature spine densities. Golgi staining of granule cells in the hippocampal dentate gyri from 12-week-old ARHGAP33 KO and WT mice. Scale bar, $10 \mu \mathrm{m}$. E. and F. Z-stacks were created, and individual spines were measured (WT, $n=33$ cells KO, $n=29$ cells; each $n=4$ mice; total spine density: $p=4.4 \times 10^{-5}$; percentage of mature spines: $p=7.7 \times 10^{-17}$; one-way ANOVA; b, c). ${ }^{*} \mathrm{p}<0.05$. The bars represent the mean value.

(Nakazawa T, et al: Nat Commun, 2016; 7: $10594^{39)}$ )

of a genetic risk factor, including decreased neurotransmission caused by a decrease in mature spines and clinically-based morphological brain abnormalities. This study also suggests that the pathophysiology of schizophrenia, including genetics and clinical features, is influenced by this gene in some patients. The odds ratio of the risk allele at ARHGAP33 (e.g., rs231228, T allele) is 1.14, which suggests a weak genetic influence that could not account for the $1 \%$ prevalence rate for schizophrenia. Schizophrenia research is often non-specific because the diagnostic criteria for schizophrenia are wide-ranging and heterogeneous ${ }^{40)}$. These criteria do not take into account the presence of auditory hallucinations, which is one of the most common symptoms of schizophrenia. Thus, it is difficult to establish differences in the pathophysiology of patients with and without auditory hallucinations. Studies on biological and psychophysiological features of schizophrenia that are not included in the diagnostic criteria can resolve the clinical features (including symptoms). Furthermore, these diagnostic limitations suggest that schizophrenia should be currently classified as a syndrome rather than a disorder.

\section{Conclusion}

The past 26 years of our research at Juntendo University was based on clinical practices and molecular biology studies. We established that the pathophysiology of schizophrenia can be influenced by genetics, altered neurotransmission, brain morphology, and clinical features. However, these results are not ready for direct translation to all patient populations, especially treatment-resistant patients. Ceaseless efforts from the JUSP study group continue to focus on schizophrenia pathophysiology in hopes of discovering new therapeutics for our patients.

\section{Acknowledgements}

I would like to acknowledge the help of JUSP members in all studies. I would like to express my gratitude to my former professors Iizuka R, Inoue R, and Nagata $T$ of Department of Psychiatry, Juntendo University Faculty of Medicine for coaching me on neuropathology, neurophysiology, and clinical psychiatry. I would like to express my sincere gratitude to professor Arai $\mathrm{H}$ of Department of 
Psychiatry, Juntendo University Faculty of Medicine for not only teaching me subjects from clinical to molecular biology in psychiatry but also for teaching me on how a good clinical researcher should be at a University.

\section{References}

1) Kane J, Honigfeld G, Singer J, Meltzer H: Clozapine for the treatment-resistant schizophrenic. A double-blind comparison with chlorpromazine. Arch Gen Psychiatry, 1988; 45: 789-796.

2) Crow TJ, Frith CD, Johnstone EC, Owens DGC, Weinberger D, Wyatt RJ: Schizophrenia and cerebral atrophy. Lancet, 1980; 1: 1129-1130.

3) Ohnuma T, Kimura M, Takahashi T, Iwamoto N, Arai H: A magnetic resonance imaging study in first-episode disorganized-type patients with schizophrenia. Psychiatry Clin Neurosci, 1997; 51: 9-15.

4) Bogerts B: The neuropathology of schizophrenic diseases: historical aspects and present knowledge. Eur Arch Psychiatry Clin Neurosci, 1999; 249 Suppl 4: 2-13

5) Bruton CJ, Crow TJ, Frith CD, Johnstone EC, Owens DG, Roberts GW: Schizophrenia and the brain: a prospective clinico-neuropathological study. Psychol Med, 1990; 20: $285-304$.

6) Ohnuma T, Augood SJ, Arai H, McKenna PJ, Emson PC: Expression of the human excitatory amino acid transporter 2 and metabotropic glutamate receptors 3 and 5 in the prefrontal cortex from normal individuals and patients with schizophrenia. Brain Res Mol Brain Res, 1998; 56: 207-217.

7) Ohnuma T, Augood SJ, Arai H, McKenna PJ, Emson PC: Measurement of GABAergic parameters in the prefrontal cortex in schizophrenia: focus on GABA content, GABA(A) receptor alpha-1 subunit messenger RNA and human GABA transporter-1 (HGAT-1) messenger RNA expression. Neuroscience, 1999; 93: 441-448.

8) Ohnuma T, Suzuki T, Arai H: Hypothesis: minimal changes in neural transmission in schizophrenia: decreased glutamatergic and GABAergic functions in the prefrontal cortex. Prog Neuropsychopharmacol Biol Psychiatry, 2005; 29: 889-894.

9) Hanzawa R, Ohnuma T, Nagai Y, et al: No association between glutathione-synthesis-related genes and Japanese schizophrenia. Psychiatry Clin Neurosci, 2011; 65: 39-46.

10) Higa M, Ohnuma T, Maeshima H, et al: Association analysis between functional polymorphism of the rs4606 SNP in the RGS2 gene and antipsychotic-induced Parkinsonism in Japanese patients with schizophrenia: results from the Juntendo University Schizophrenia Projects (JUSP). Neurosci Lett, 2010; 469: 55-59.

11) Higashi M, Higashi S, Ohnuma T, Shibata N, Sakai $Y$, Arai H: Genetic analysis of the TrkB gene and schizophrenia in the Japanese population: Juntendo University Schizophrenia Projects (JUSP). Neurosci Lett, 2007; 425: 1-5.

12) Higashi S, Ohnuma T, Shibata N, Higashi M, Matsubara Y, Arai H: No genetic association between tryptophan hydroxylase 2 gene polymorphisms and Japanese schizophrenia. Psychiatr Genet, 2007; 17: 123.
13) Hotta $Y$, Ohnuma T, Hanzawa R, et al: Association study between Disrupted-in-Schizophrenia-1 (DISC1) and Japanese patients with treatment-resistant schizophrenia (TRS). Prog Neuropsychopharmacol Biol Psychiatry, 2011; 35: 636-639.

14) Kawashima R, Ohnuma T, Shibata N, Arai H: No genetic association between postsynaptic density-95 gene polymorphisms and schizophrenia. Neurosci Lett, 2006; 400: $168-171$

15) Nagai $Y$, Ohnuma T, Karibe J, et al: No genetic association between the SLC1A2 gene and Japanese patients with schizophrenia. Neurosci Lett, 2009; 463: 223-227.

16) Nozawa M, Ohnuma $T$, Matsubara $Y$, et al: The relationship between the response of clinical symptoms and plasma olanzapine concentration, based on pharmacogenetics: Juntendo University Schizophrenia Projects (JUSP). Ther Drug Monit, 2008; 30: 35-40.

17) Ohnuma $T$, Shibata N, Maeshima $\mathrm{H}$, et al: Association analysis of glycine- and serine-related genes in a Japanese population of patients with schizophrenia. Prog Neuropsychopharmacol Biol Psychiatry, 2009; 33: 511518.

18) Takebayashi Y, Ohnuma T, Hanzawa R, et al: No genetic association between SLC7A10 and Japanese patients with schizophrenia. Prog Neuropsychopharmacol Biol Psychiatry, 2011; 35: 1965-1968.

19) Kitazawa M, Ohnuma T, Takebayashi $Y$, et al: No associations found between the genes situated at $6 \mathrm{p} 22.1$, HIST1H2BJ, PRSS16, and PGBD1 in Japanese patients diagnosed with schizophrenia. Am J Med Genet B Neuropsychiatr Genet, 2012; 159B: 456-464.

20) Ohi K, Hashimoto R, Yasuda Y, et al: Functional genetic variation at the NRGN gene and schizophrenia: evidence from a gene-based case-control study and gene expression analysis. Am J Med Genet B Neuropsychiatr Genet, 2012; 159B: 405-413.

21) Ohi K, Hashimoto R, Yasuda Y, et al: Association study of the G72 gene with schizophrenia in a Japanese population: a multicenter study. Schizophr Res, 2009; 109: 80-85.

22) Ohnuma T, Shibata N, Baba H, et al: No association between DAO and schizophrenia in a Japanese patient population: a multicenter replication study. Schizophr Res, 2010; 118: 300-302.

23) Arinami T, Ohtsuki T, Ishiguro H, et al: Genomewide high-density SNP linkage analysis of 236 Japanese families supports the existence of schizophrenia susceptibility loci on chromosomes 1p, 14q, and 20p. Am J Hum Genet, 2005; 77: 937-944.

24) Ohnuma T, Arai H: Genetic or psychogenic? A case study of "folie à quatre" including twins. Case Rep Psychiatry, 2015; 2015: 983212.

25) Hatano T, Ohnuma T, Sakai Y, et al: Plasma alanine levels increase in patients with schizophrenia as their clinical symptoms improve-results from the Juntendo University Schizophrenia Projects (JUSP). Psychiatry Res, 2010; 177: 27-31.

26) Maeshima H, Ohnuma T, Sakai Y, et al: Increased plasma glutamate by antipsychotic medication and its relationship to glutaminase 1 and 2 genotypes in schizophrenia - Juntendo University Schizophrenia Projects (JUSP). Prog Neuropsychopharmacol Biol Psychiatry, 2007; 31: 1410-1418.

27) Ohnuma T, Sakai Y, Maeshima H, et al: Changes in 
plasma glycine, L-serine, and D-serine levels in patients with schizophrenia as their clinical symptoms improve: results from the Juntendo University Schizophrenia Projects (JUSP). Prog Neuropsychopharmacol Biol Psychiatry, 2008; 32: 1905-1912.

28) Ohnuma T, Sakai Y, Maeshima H, et al: No correlation between plasma NMDA-related glutamatergic amino acid levels and cognitive function in medicated patients with schizophrenia. Int J Psychiatry Med, 2012; 44: 17-27.

29) Ohnuma T, Arai H: Significance of NMDA receptorrelated glutamatergic amino acid levels in peripheral blood of patients with schizophrenia. Prog Neuropsychopharmacol Biol Psychiatry, 2011; 35: 29-39.

30) Arai M, Yuzawa H, Nohara I, et al: Enhanced carbonyl stress in a subpopulation of schizophrenia. Arch Gen Psychiatry, 2010; 67: 589-597.

31) Katsuta N, Ohnuma T, Maeshima H, et al: Significance of measurements of peripheral carbonyl stress markers in a cross-sectional and longitudinal study in patients with acute-stage schizophrenia. Schizophr Bull, 2014; 40: 1366-1373.

32) Takeda M, Ohnuma T, Takeuchi M, et al: Altered serum glyceraldehyde-derived advanced glycation end product (AGE) and soluble AGE receptor levels indicate carbonyl stress in patients with schizophrenia. Neurosci Lett, 2015; 593: 51-55.

33) Ikeda M, Aleksic B, Yamada K, et al: Genetic evidence for association between NOTCH4 and schizophrenia supported by a GWAS follow-up study in a Japanese population. Mol Psychiatry, 2013; 18: 636-638.

34) International-Schizophrenia-Consortium: Rare chromosomal deletions and duplications increase risk of schizophrenia. Nature, 2008; 455: 237-241.

35) Stefansson H, Rujescu D, Cichon S, et al: Large recurrent microdeletions associated with schizophrenia. Nature, 2008; 455: 232-236.

36) Bassett AS, Marshall CR, Lionel AC, Chow EW, Scherer SW: Copy number variations and risk for schizophrenia in 22q11.2 deletion syndrome. Hum Mol Genet, 2008; 17: 4045-4053.

37) Higashiyama R, Ohnuma T, Takebayashi $\mathrm{Y}$, et al: Association of copy number polymorphisms at the promoter and translated region of COMT with Japanese patients with schizophrenia. Am J Med Genet B Neuropsychiatr Genet, 2016; 171: 447-457.

38) Nakamura T, Ohnuma T, Hanzawa R, et al: Associations of common copy number variants in glutathione S-transferase mu 1 and D-dopachrome tautomeraselike protein genes with risk of schizophrenia in a Japanese population. Am J Med Genet B Neuropsychiatr Genet, 2015; 168: 630-636.

39) Nakazawa T, Hashimoto R, Sakoori K, et al: Emerging roles of ARHGAP33 in intracellular trafficking of TrkB and pathophysiology of neuropsychiatric disorders. Nat Commun, 2016; 7: 10594.

40) First MB, Spitzer RL, Gibbon M, et al: Structured Clinical Interview for DSM-IV Axis I Disorders. Biometrics Research Department, New York State Psychiatric Institute, New York, 1995. 